\title{
Protective effects of SOCS3 overexpression in high glucose-induced lung epithelial cell injury through the JAK2/STAT3 pathway
}

\author{
WEI-NA DUAN, ZHONG-YUAN XIA, MIN LIU, QIAN SUN, \\ SHAO-QING LEI, XIAO-JING WU, QING-TAO MENG and YAN LENG
}

Department of Anesthesiology, Renmin Hospital of Wuhan University, Wuhan, Hubei 430060, P.R. China

Received August 2, 2016; Accepted May 5, 2017

DOI: $10.3892 / \mathrm{mmr} .2017 .6941$

\begin{abstract}
Previous studies have suggested that the Janus kinase (JAK)/signal transducers and activators of transcription (STAT) pathway is involved in hyperglycemia-induced lung injury. The present study aimed to investigate the roles of suppressor of cytokine signaling3 (SOCS3) in the regulation of JAK2/STAT3 activation following high glucose (HG) treatment in A549 human pulmonary epithelial cells. Cell viability was evaluated using Cell Counting Kit- 8 and lactate dehydrogenase assays. HG-induced inflammatory injury in A549 cells was assessed through the evaluation of interleukin-6 (IL-6) and tumor necrosis factor- $\alpha(\mathrm{TNF}-\alpha)$ levels using ELISA. The protein expression levels of SOCS3, JAK2, STAT3, phosphorylated (p)-JAK2 and p-STAT3 were determined using western blot analysis. Cellular viability was significantly decreased, whereas IL-6 and TNF- $\alpha$ levels were significantly increased, following HG stimulation of A549 cells. In addition, the protein levels of SOCS3, p-JAK2 and p-STAT3 were significantly increased in HG-treated cells. Treatment with the JAK2/STAT3 inhibitor tyrphostin AG490, or SOCS3 overexpression, appeared to prevent the HG-induced alterations in protein expression. Furthermore, cellular viability was enhanced, whereas the levels of proinflammatory cytokines were suppressed. These finding suggested the involvement of the SOCS3/JAK2/STAT3 signaling pathway in HG-induced responses in lung cells. Therefore, it may be hypothesized that the inhibition of the JAK2/STAT3 pathway through SOCS3 overexpression may prevent hyperglycemia-induced lung injury, and may have therapeutic potential for the treatment of patients with diabetic lung injury.
\end{abstract}

Correspondence to: Professor Zhong-Yuan Xia, Department of Anesthesiology, Renmin Hospital of Wuhan University, 238 Jiefang Road, Wuhan, Hubei 430060, P.R. China

E-mail: xiazhongyuan2005@aliyun.com

Key words: suppressor of cytokine signaling 3, high glucose, Janus kinase2/signal transducers and activators of transcription 3 , human lung epithelial cells, injury

\section{Introduction}

Diabetes mellitus is a metabolic disease with an increasing prevalence worldwide (1). Micro- and macrovascular disorders with debilitating consequences for several organs are prevalent in patients with diabetes (2). The alveolar-capillary network in the lungs is a large microvascular unit and can be affected by microangiopathy (3). Diabetes has detrimental effects on retinal and glomerular microvasculature (4); however, pulmonary diabetic microangiopathy has not received considerable clinical attention, as due to the large reserve of the lung microvasculature, it is possible that substantial pulmonary dysfunction may occur without the appearance of dyspnea (3). Therefore, the specific molecular mechanisms underlying the development of metabolic defects in the lungs have yet to be elucidated.

Previous studies have suggested that inflammation may serve crucial roles in the pathogenesis of diabetes $(5,6)$. Therefore, the inhibition of inflammation has potential as a novel therapeutic strategy for the treatment of patients with diabetes (6). Several proinflammatory cytokines have been implicated in the development of diabetic lung injury (7-9). The Janus kinase (JAK)/signal transducers and activators of transcription (STAT) intracellular signaling pathway is activated in response to cytokine stimulation and relays biological signals to target cells (10). The JAK/STAT pathway has been involved in the regulation of several genes involved in cell proliferation, inflammation and fibrosis. In addition, the JAK/STAT pathway has been implicated in hyperglycemia-induced nephropathy in patients with diabetes $(11,12)$.

The JAK/STAT pathway is negatively regulated via various mechanisms, including the suppressor of cytokine signaling (SOCS) proteins, which have been identified as negative feedback regulators of cytokine signaling, and have been reported to be involved in the regulation of inflammatory responses (13-17). The mammalian cytokine-inducible SH2-containing protein (CIS)/SOCS family consists of 8 members, including CIS and SOCS1-7 (18). CIS/SOCS proteins are characterized by a central $\mathrm{SH} 2$ domain, an amino-terminal domain of variable length and sequence and a carboxy-terminal SOCS box (19). The presence of SOCS3 has been demonstrated in the lungs (14). The SH2 domain of 
SOCS3 does not have a high affinity for the activation loop of JAKs; however, the kinase inhibitory region of SOCS3 has a higher affinity for the kinase domain of JAK2 compared with that of SOCS1 (20). Since SOCS3 can bind to interleukin (IL)-12 receptor, which can activate STAT3, SOCS3 can inhibit the IL-12-mediated activation of STAT3 (21).

Increasing evidence indicates that SOCS proteins may be involved in the development of diabetes and disease-associated complications (22). In addition, SOCS-modulating properties have been attributed to pharmacological agents that are currently used for the treatment of diabetes (17). The present study aimed to investigate the function of SOCS3 in lung cells and to explore the implication of the JAK2/STAT3 signaling pathway in the molecular mechanisms underlying the effects of SOCS3 in hyperglycemia-induced lung injury.

\section{Materials and methods}

Materials. A549 human lung epithelial cells were purchased from American Type Culture Collection (Manassas, VA, USA). Dulbecco's modified Eagle's medium (DMEM) was purchased from Hyclone (GE Healthcare Life Sciences, Logan, UT, USA) and fetal bovine serum (FBS) was obtained from Gibco (Thermo Fisher Scientific, Inc., Waltham, MA, USA). Phosphate-buffered saline (PBS), and $0.25 \%$ trypsin with $0.02 \%$ EDTA were obtained from Hyclone (GE Healthcare Life Sciences). D-glucose and D-mannitol were from Sinopharm Chemical Reagent Co., Ltd. (Shanghai, China), and tyrphostin AG490 was from Sigma-Aldrich (Merck KGaA, Darmstadt, Germany). Empty vector plasmid pcDNA3.1 and the expression vector pcDNA3.1-SOCS3 were synthesized by Shanghai GenePharma Co., Ltd. (Shanghai, China). Attractene transfection reagent was obtained from Qiagen, Inc. (Valencia, CA, USA). The following antibodies were used: Anti-JAK2 (1:1,000; catalog no. 3230), anti-STAT3 (1:1,000; catalog no. 4904), anti-phosphorylated (p)-JAK2 (1:1,000; catalog no. 3776), anti-p-STAT3 (1:1,000; catalog no. 9145) and anti-SOCS3 polyclonal antibodies (1:1,000; catalog no. 2932) (all from Cell Signaling Technology, Inc., Danvers, MA, USA). The $\beta$-actin antibody (1:1,000; catalog no. 4970; Cell Signaling Technology, Inc.) served as the loading control. The proteins were visualized using secondary antibodies: Goat anti-rabbit polyclonal IgG (1:10,000; catalog no. 926-32221; LI-COR Biosciences, Lincoln, NE, USA). The following kits were used: Cell Counting Kit-8 (CCK-8; Dojindo Molecular Technologies, Inc., Kumamoto, Japan), lactate dehydrogenase (LDH) cytotoxicity kit, IL-6 (catalog no. H052; Nanjing Jiancheng Bioengineering Institute, Nanjing, China) and tumor necrosis factor (TNF)- $\alpha$ ELISA kits (catalog no. H007; Nanjing Jiancheng Bioengineering Institute, Nanjing, China), and bicinchoninic acid (BCA) protein assay kit (Beyotime Institute of Biotechnology, Haimen, China).

Cell culture. A549 cells were cultured in DMEM containing $5.5 \mathrm{mM}$ glucose, supplemented with $10 \%$ FBS. Following $24 \mathrm{~h}$, cells were subcultured using $0.25 \%$ trypsin with $0.02 \%$ EDTA following washing with PBS twice, when they reached $70-80 \%$ confluence. The following treatment groups were used: Normal glucose (NG) group, fresh $5.5 \mathrm{mM}$ glucose was added to the medium; high glucose (HG) group, $25 \mathrm{mM}$ glucose was added to the medium; and high osmosis (OG) group, $5.5 \mathrm{mM}$ glucose and $19.5 \mathrm{mM}$ D-mannitol were added to the medium. All cells were incubated at $37^{\circ} \mathrm{C}$ in a5\% $\mathrm{CO}_{2}$ atmosphere for $48 \mathrm{~h}$ (23).

When the A549 cells reached 70-80\% confluence they were cultured in DMEM containing $5.5 \mathrm{mM}$ glucose without FBS for $24 \mathrm{~h}$. Subsequently, they were treated with $10 \mu \mathrm{mol} / \mathrm{l}$ tyrphostin AG490 in serum-free DMEM containing $25 \mathrm{mM}$ glucose (HG+AG490 group) and incubated at $37^{\circ} \mathrm{C}$ in a $5 \%$ $\mathrm{CO}_{2}$ atmosphere for $24 \mathrm{~h}$. Cells were then collected for subsequent experiments.

Plasmid transfection. A549 cells were seeded at a density of $2 \times 10^{5}$ cells $/ \mathrm{ml}$ in DMEM supplemented with FBS $24 \mathrm{~h}$ prior to transfection. Cells were $40-80 \%$ confluent on the day of transfection and were treated according to the manufacturer's protocol. Cells were transfected, using Attractene, with empty vector control (HG+SOCS3 - group) or with pcDNA3.1-SOCS3 expression vector $\left(\mathrm{HG}+\mathrm{SOCS}^{+}\right.$group) and incubated in serum-free medium for $6 \mathrm{~h}$. Subsequently, the medium was replaced with fresh medium containing $5.5 \mathrm{mM}$ glucose and cells were cultured for $24 \mathrm{~h}$. Then, the medium was replaced with $25 \mathrm{mM}$ glucose-containing medium and cells were incubated for an additional $24 \mathrm{~h}$.

Cell viability assay. A total of $100 \mu 1$ adherent A549 cells from each experimental group were cultured in 96-well plates $\left(1 \times 10^{4}\right.$ cells/well), and the supernatant was collected for the LDH toxicity assay. The assay was performed according to the manufacturer's protocol and the absorbance of each sample was measured at $450 \mathrm{~nm}$ using a microplate reader. For the CCK-8 assay, the medium was removed and cells were washed twice with PBS. Fresh medium and $10 \mu \mathrm{l}$ CCK-8 solution were added to each well and cells were incubated for $2 \mathrm{~h}$ at $37^{\circ} \mathrm{C}$ in $5 \% \mathrm{CO}_{2}$. Subsequently, the absorbance of each sample was measured of $450 \mathrm{~nm}$ using a microplate reader. The mean optical density (OD) values of six randomly selected wells from each treatment group were used as an index of cell viability.

Proinflammatory cytokine ELISA. A549 cells were seeded in a 6 -well plate at a density of $2 \times 10^{5}$ cells $/ \mathrm{ml}$ and stimulated with different conditions. Following treatment, cells were washed three times with PBS and their IL- 6 and TNF- $\alpha$ contents were measured using ELISA kits, according to the manufacturer's protocol.

Western blot analysis. Cells were rinsed twice with PBS and dissolved in SDS sample loading buffer. Total proteins were extracted using a radioimmunoprepitation assay lysis buffer (Beyotime Institute of Biotechnology). Protein concentrations were determined using a BCA assay. Equal amounts of extracted protein samples $(30 \mu \mathrm{g})$ were separated by $10 \%$ SDS-PAGE and transferred onto a polyvinylidenedifluoride membrane. The membrane was blocked with $5 \%$ bovine serum albumin (BSA; Beyotime Institute of Biotechnology) at room temperature for $2 \mathrm{~h}$ under agitation to prevent non-specific antibody binding. The membranes were then incubated with the following primary antibodies at $4^{\circ} \mathrm{C}$ for $12 \mathrm{~h}$ : Anti-STAT3, anti-SOCS3, anti-JAK2, anti-p-JAK2 and anti-p-STAT3, and anti- $\beta$-actin. 

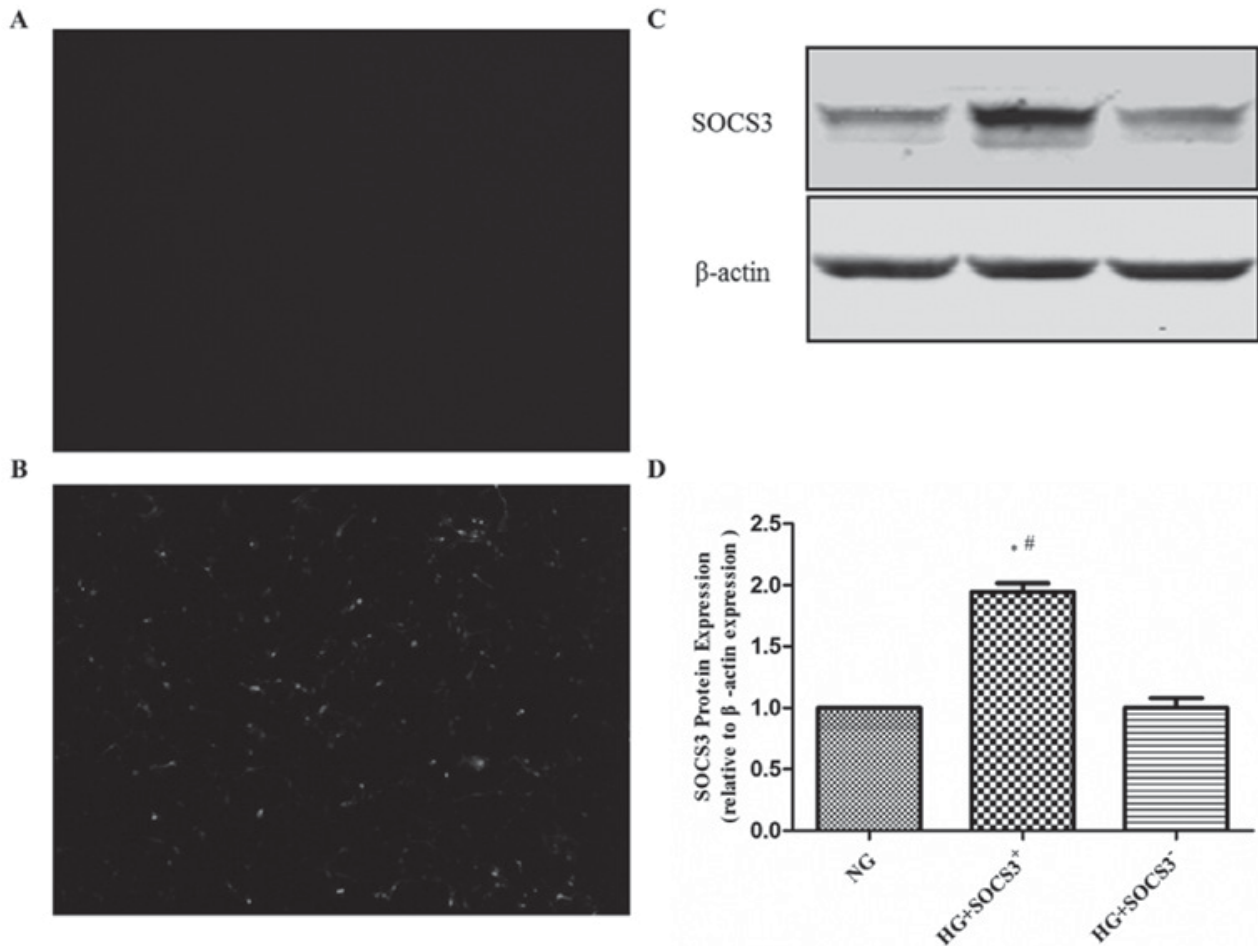

Figure 1. Successful overexpression of SOCS3 in human A549 pulmonary epithelial cells. Representative photomicrographs demonstrating successful transfection of A549 cells with (A) empty vector ( $\mathrm{NG+SOCS3}{ }^{-}$) and (B) pcDNA3.1-SCOS3 expression vector (NG+SOCS3 $3^{+}$. Magnification, $\mathrm{x} 200$. (C) Representative blot demonstrating SOCS3 expression in A549 cells following transfection. $\beta$-actin was used as the loading control. (D) Blots were semi-quantified using densitometry. Data are expressed as the mean \pm standard error of the mean of 5 independent experiments. ${ }^{\text {P }}<0.05$ vs. NG group; ${ }^{~} \mathrm{P}<0.05$ vs. NG+SOCS3 group. SOCS, suppressor of cytokine signaling; NG, normal glucose.

Subsequently, the membrane was rinsed with PBS and incubated with goat anti-rabbit horseradish peroxidase-conjugated secondary antibody for $1 \mathrm{~h}$ at room temperature. The protein bands were visualized by enhanced chemiluminescence using an Odyssey Infrared Imaging system (LI-COR Biosciences), and blots were semi-quantified by densitometry using Quantity One software version 4.6.2 (Bio-Rad Laboratories, Inc., Hercules, CA, USA).

Statistical analysis. Data are presented as the mean \pm standard error of the mean. Statistical analysis was performed using GraphPad Prism software version 5.0 (GraphPad Software, Inc., La Jolla, CA, USA). The statistical significance of the differences between groups was evaluated using an unpaired Student's t-test for pair-wise comparisons, or a one-way analysis of variance followed by the Tukey post hoc test for multiple comparisons. $\mathrm{P}<0.05$ was considered to indicate a statistically significant difference.

\section{Results}

SOCS3 is overexpressed in A549 cells following transfection. To assess the effects of SOCS3 activation in hyperglycemia-induced lung injury, human A549 lung epithelial cells were transfected with pcDNA3.1-SCOS3 expression vector expressing green fluorescence or empty vector control expressing no fluorescence. As presented in Fig. 1A and B, intense green fluorescence was observed in pcDNA3.1-SCOS3-transfected cells compared with control vector-transfected cells. Furthermore, western blot analysis demonstrated that the protein expression of SOCS3 was significantly increased by transfection with the SOCS3 overexpression plasmid compared with the empty vector-transfected group (Fig. 1C and D).

Morphological alterations following SOCS3 overexpression in HG-treated A549 cells. The morphology of A549 cells from the various treatment groups was observed under an inverted microscope. A549 cells cultured in normal control medium (NG group) exhibited shuttle-like shapes, and long and fine cell bodies (Fig. 2A). However, exposure to HG markedly altered the morphology of the cells in the HG group, which exhibited shorter and less extended cell bodies, and the cells were dead and lysed with more cell fragments (Fig. 2B), thus suggesting that $\mathrm{HG}$ exposure may induce apoptotic morphological characteristics in A549 cells. When exposed to high osmotic pressure, the cells exhibited fusiform shapes with failed outgrowth of processes, whereas cell loss was also observed (Fig. 2C). Treatment with tyrphostin AG490 appeared to attenuate the morphological changes in HG-treated cells, and to increase their density (Fig. 2D). Cell morphology appeared to be similar between cells in the HG and $\mathrm{HG}+\mathrm{SOCS}^{-}$groups (Fig. 2E); whereas SOCS3 overexpression appeared to produce similar effects in HG-exposed cells (Fig. 2F) appeared to attenuate the morphological changes caused by HG.

HG exposure suppresses the viability of A549 cells. A CCK-8 assay was used to evaluate the viability of A549 cells from the various treatment groups in vitro. As presented in Fig. 3A, the viability of cells exposed to $\mathrm{HG}$ was significantly decreased 

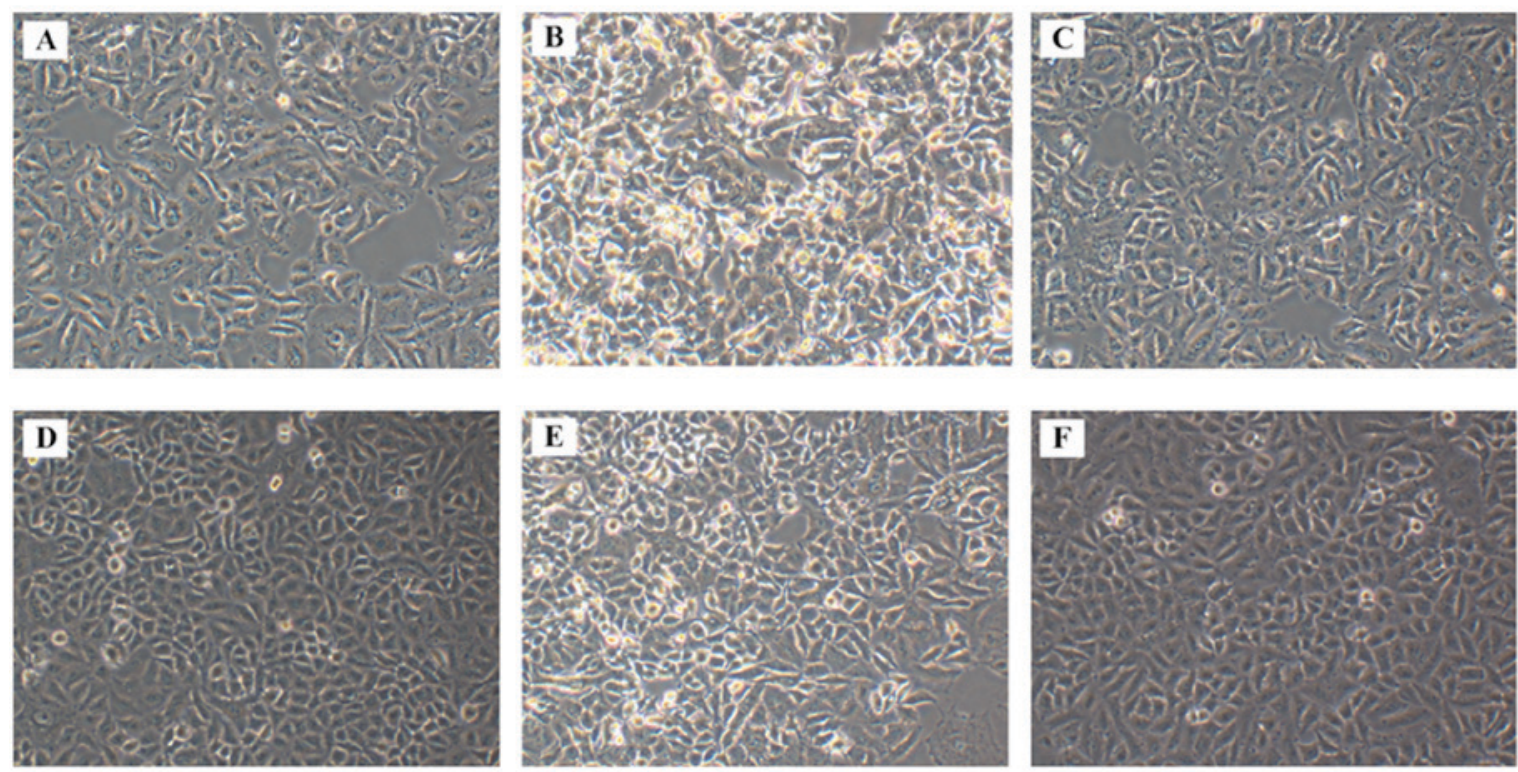

Figure 2. Morphological alterations of human A549 pulmonary epithelial cells following HG exposure. Representative photomicrographs of cells cultured in (A) normal glucose conditions, (B) HG conditions, (C) hyperosmotic conditions, (D) HG conditions + tyrphostin AG490, (E) HG conditions + empty vector and (F) HG conditions + pcDNA3.1-SOCS3 expression vector. Photomicrographs were captured using an inverted microscope. Magnification, x200. HG, high glucose; SOCS3, suppressor of cytokine signaling.
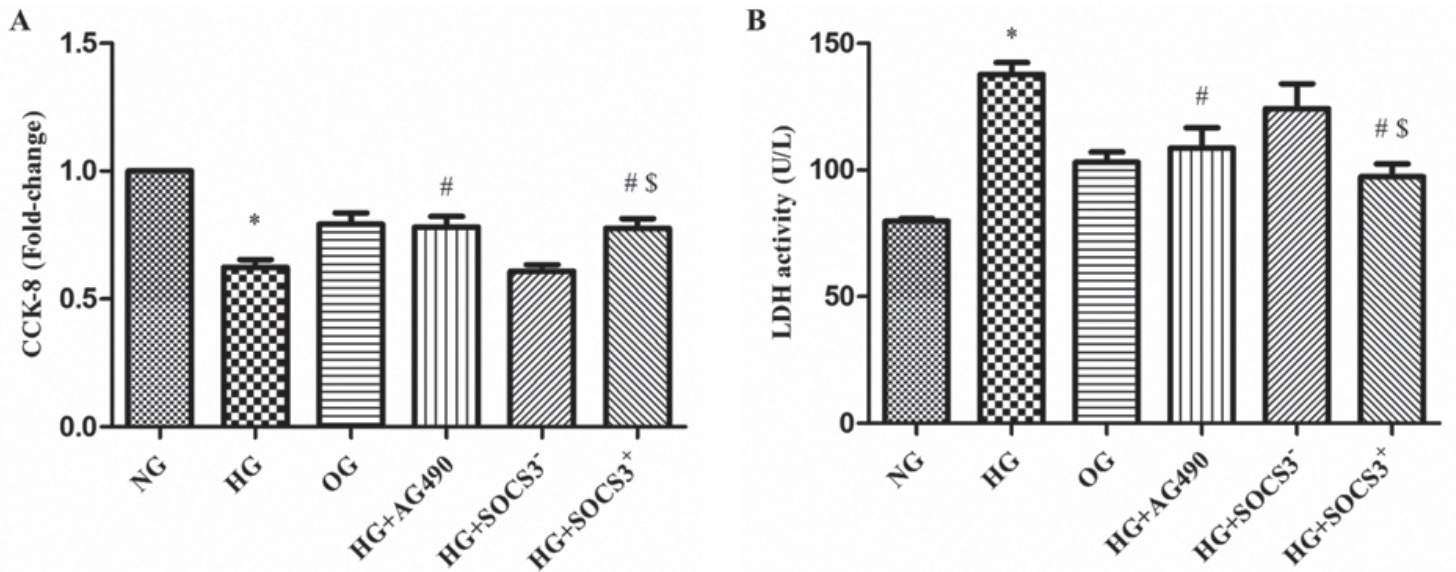

Figure 3. Viability of human A549 pulmonary epithelial cells following various treatments evaluated using. (A) Cell Counting Kit-8 and (B) LDH cytotoxicity assays. Cells were cultured under NG, HG and OG conditions, with HG + tyrphostin AG490, and with HG + pcDNA3.1-SOCS3 expression vector. Data are expressed as the mean \pm standard error of the mean of six independent measurements. ${ }^{*} \mathrm{P}<0.05$ vs. NG group; ${ }^{\text {}} \mathrm{P}<0.05$ vs. $\mathrm{HG}$ group; ${ }^{\circ} \mathrm{P}<0.05$ vs. $\mathrm{HG}+\mathrm{SOCS} 3$ group. LDH, lactate dehydrogenase; NG, normal glucose; HG, high glucose; OG, hyperosmotic group; SOCS3, suppressor of cytokine signaling 3.

compared with the NG group $(\mathrm{P}<0.05)$. Notably, the viability of cells in the $\mathrm{HG}+\mathrm{AG} 490$ and $\mathrm{HG}+\mathrm{SOCS}^{+}$groups was significantly enhanced compared with cells in the HG group $\left(\mathrm{P}<0.05\right.$; Fig. 3A). In addition, cells in the $\mathrm{HG}+\mathrm{SOCS}^{+}$group exhibited increased viability compared with cells in the $\mathrm{HG}+\mathrm{SOCS}^{-}$group $(\mathrm{P}<0.05$; Fig. $3 \mathrm{~A})$.

An LDH cytotoxicity assay was also used to evaluate cell viability. The present results demonstrated that $\mathrm{LDH}$ activity was significantly increased in HG-treated cells compared with the NG group ( $\mathrm{P}<0.05$; Fig. 3B). Conversely, $\mathrm{LDH}$ activity in cells from the $\mathrm{HG}+\mathrm{AG} 490$ and $\mathrm{HG}+\mathrm{SOCS}{ }^{+}$groups was significantly suppressed compared with the HG group, indicating an increase in cell viability $(\mathrm{P}<0.05$; Fig. 3B). Furthermore, the viability of $\mathrm{HG}+\mathrm{SOCS}^{+}$cells was significantly enhanced compared with $\mathrm{HG}+\mathrm{SOCS}^{-}$cells $(\mathrm{P}<0.05$; Fig. 3B). Notably, hyperosmolarity did not appear to exert an effect on A549 cell viability (OG group; Fig. 3A and B).

$H G$ induces the expression of IL- 6 and TNF- $\alpha$ in A549 cells. As presented in Fig. 4, IL-6 levels were increased by $>2$-fold in HG-treated cells compared with in NG cells $(\mathrm{P}<0.05$; Fig. 4A). In addition, TNF- $\alpha$ levels were increased by 3 -fold following HG exposure ( $\mathrm{P}<0.05$; Fig. 4B). Notably, IL- 6 and TNF- $\alpha$ levels in cells from the $\mathrm{HG}+\mathrm{AG} 490$ and $\mathrm{HG}+\mathrm{SOCS}^{+}$groups were significantly decreased compared with in cells from the HG group $(\mathrm{P}<0.05)$. Furthermore, IL-6 and TNF- $\alpha$ levels were significantly increased in the $\mathrm{HG}+\mathrm{SOCS}^{-}$group compared with in $\mathrm{HG}+\mathrm{SOCS}^{+}$cells $(\mathrm{P}<0.05$; Fig. $4 \mathrm{~A}$ and $\mathrm{B})$. 

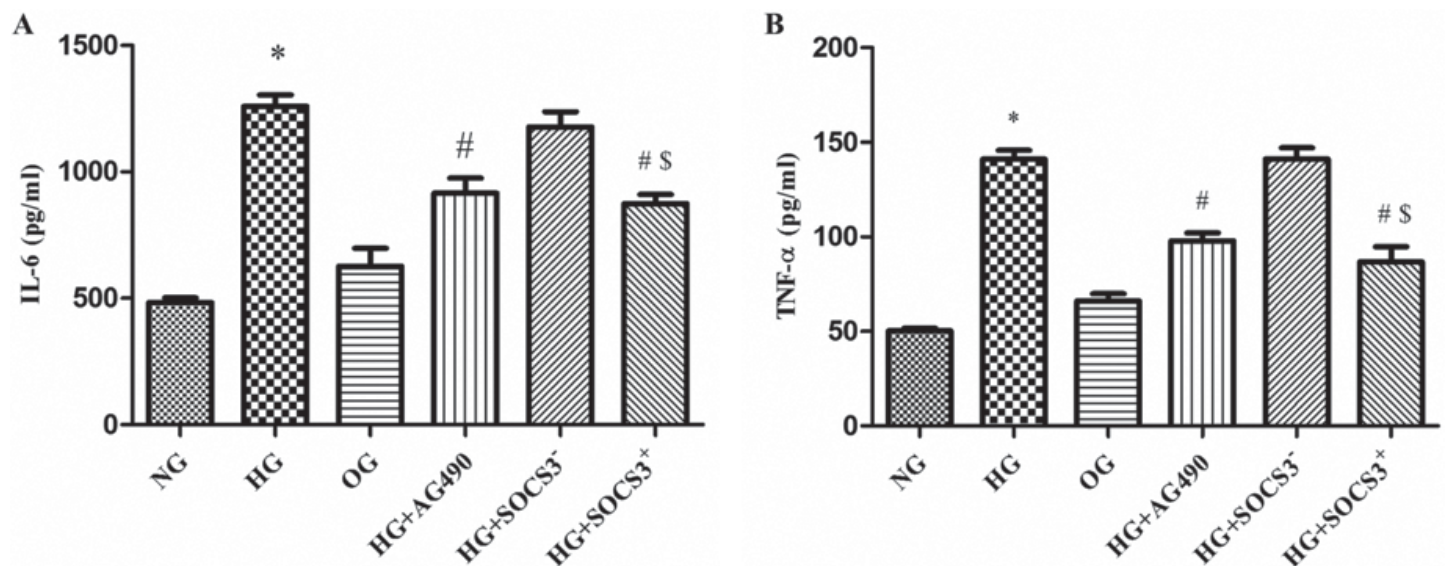

Figure 4. Proinflammatory cytokine levels in human A549 pulmonary epithelial cells following various treatments. (A) IL-6 and (B) TNF- $\alpha$ levels were measured using ELISA. Cells were cultured under NG, HG and OG conditions, with HG + tyrphostin AG490, and with HG + pcDNA3.1-SOCS3 expression vector. Data are expressed as the mean \pm standard error of the mean of six independent measurements. ${ }^{*} \mathrm{P}<0.05$ vs. NG group; ${ }^{\text {}} \mathrm{P}<0.05$ vs. HG group; ${ }^{\text {P }}<0.05$ vs. HG + SOCS3- group. IL, interleukin; TNF, tumor necrosis factor; NG, normal glucose; HG, high glucose; OG, hyperosmotic group; SOCS3, suppressor of cytokine signaling 3 .
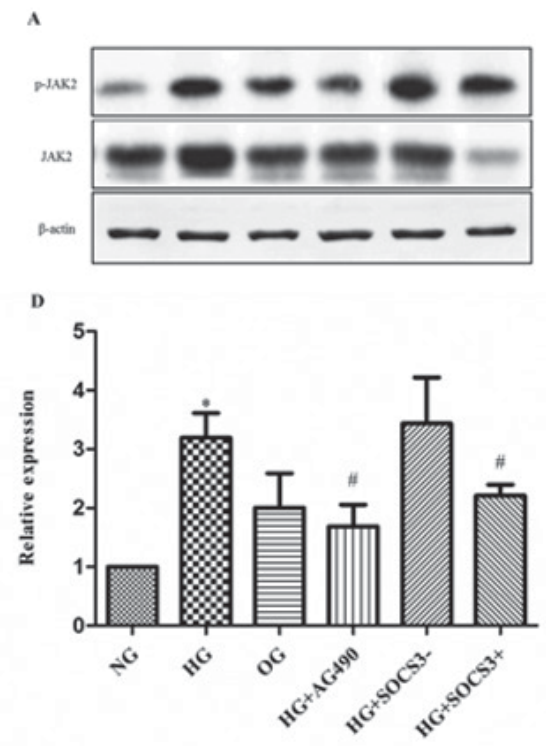
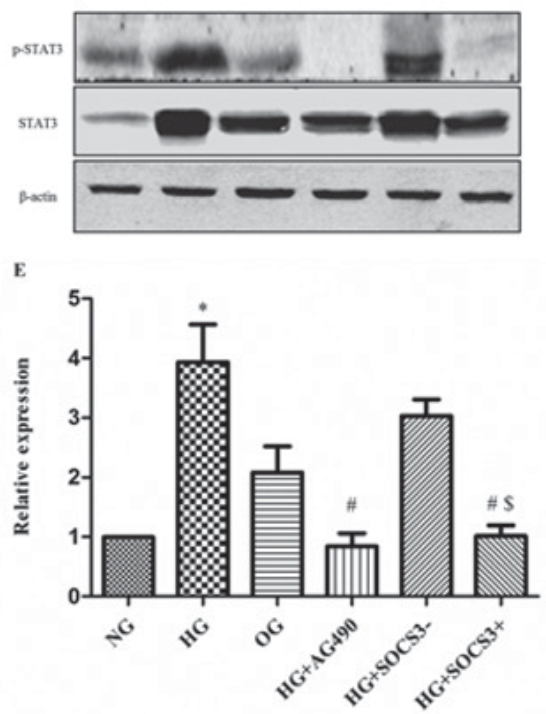

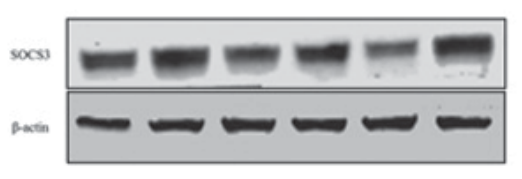

$\mathbf{F}$

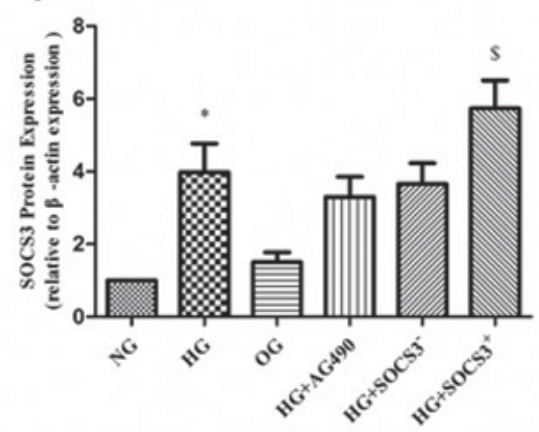

Figure 5. Protein expression levels of p-JAK2, p-STAT3 and SOCS3 in human A549 pulmonary epithelial following various treatments. Protein expression levels of (A) p-JAK2 and total JAK2, (B) p-STAT3 and total STAT3 and (C) SOCS3, were detected using western blot analysis. $\beta$-actin was used as the loading control. Blots were semi-quantified using densitometry. Densitometric analysis of (D) p-JAK2, (E) p-STAT3 and (F) SOCS3. Cells were cultured under NG, HG and OG conditions, with HG + tyrphostin AG490, and with HG + pcDNA3.1-SOCS3 expression vector. Data areexpressed as the mean \pm standard error of the mean of 10 independent experiments. " $\mathrm{P}<0.05$ vs. NG group; ${ }^{\mathrm{P}} \mathrm{P}<0.05$ vs. HG group; ${ }^{\mathrm{P}} \mathrm{P}<0.05$ vs.HG + SOCS3 group. JAK, Janus kinase; NG, normal glucose; HG, high glucose; OG, hyperosmotic group; SOCS3, suppressor of cytokine signaling 3; p-, phosphorylated; STAT, signal transducers and activators of transcription.

Effects of SOCS3 overexpression on the expression of JAK2/STAT3 pathway proteins. As demonstrated in Fig. 5, p-JAK2 and p-STAT3 densitometric units were normalized to that of total JAK2 and STAT3 respectively. The protein expression levels of p-JAK2 and p-STAT3 were significantly upregulated in HG cells compared with in cells from the NG group $(\mathrm{P}<0.05)$. Following treatment of HG-exposed cells with the JAK2/STAT3 signaling pathway inhibitor tyrphostin AG490, the protein levels of, p-JAK2 and p-STAT3 were significantly downregulated compared with the HG group $(\mathrm{P}<0.05$; Fig. 5). In addition, SOCS3 protein expression was enhanced in A549 cells following exposure to HG compared with the NG group $(\mathrm{P}<0.05 ;$ Fig. 5). To investigate the putative regulatory effects of SOCS3 on the JAK2/STAT3 pathway, SOCS3 was overexpressed in A549 cells by plasmid transfection. The results revealed that SOCS3 overexpression significantly inhibited HG-induced upregulation of both phosphorylated JAK2 and STAT3 proteins compared with the control vector-transfected groups $(\mathrm{P}<0.05$; Fig. 5). Notably, the hyperosmolarity control did not exert an effect on the expression of JAK2/STAT3 pathway proteins in A549 cells (Fig. 5).

\section{Discussion}

The present study demonstrated that the SOCS3/JAK2/STAT3 pathway was activated in HG-treated A549 lung epithelial 
cells. Previous studies reported that HG exposure enhanced the phosphorylation of proteins of the JAK/STAT pathway in human renal tubular epithelial and glomerular mesangial cells (24-26). Similarly, in the present study, treatment of pulmonary epithelial cells with HG resulted in the significant increase of p-JAK2 and p-STAT3 protein levels. In addition, $\mathrm{HG}$ exposure was revealed to upregulate the protein expression of SOCS3 in A549 cells. Notably, SOCS3 overexpression was demonstrated to attenuate the $\mathrm{HG}$-induced increases in p-JAK2 and p-STAT3, showing that SOCS3, which are negative regulators of the JAK2/STAT3 signaling pathway, are involved in hyperglycemia-induced cell responses during the development of diabetic lung injury. It has also been suggested that modulation of this pathway may prevent lung complications of diabetes.

The rapid worldwide increase in the prevalence of type 2 diabetes and diabetic lung injury has become a serious public health concern (1). Dyspnea upon exertion in patients with diabetes is often mistakenly associated with cardiovascular diseases and/or physical unfitness (3). Accumulating evidence suggests that inflammation may serve a crucial role during the pathogenesis of type 2 diabetes, thus linking diabetes to various common comorbidities that also involve inflammatory mechanisms $(7,9)$. The SOCS, JAK and Src families of kinases have been implicated in systemic responses to hyperglycemia $(10,27-29)$; however, their roles in inflammatory responses in the lungs have yet to be elucidated.

Previous studies have reported that SOCS3 expression is induced by various stimuli, including cytokines, Toll-like receptor ligands, bacteria and immune complexes $(30,31)$. In addition, SOCS3 was demonstrated to be activated by lipopolysaccharide in neutrophils and macrophages (32). The functions of SOCS3 in the lung have previously been investigated. However, the role of the SOCS3/JAK2/STAT3 signaling pathway in diabetes-induced lung injury has not yet to be elucidated. The present study demonstrated that SOCS3 may be involved in the regulation of JAK2/STAT3-mediated signaling in HG-exposed A549 cells. To the best of our knowledge, this is the first time that SOCS3 has been associated with the modulation of JAK2/STAT3 signalingin an in vitro model of diabetic lung injury.

During the pathogenesis of diabetic lung injury, hyperglycemia triggers several intracellular processes in lung cells, including the generation of reactive oxygen species, the activation of protein kinase $\mathrm{C}$ and of various proinflammatory cytokines (8). TNF- $\alpha$ and IL- 6 are proinflammatory cytokines that have been revealed to be upregulated following exposure to HG (33). In addition, the prolonged increase in IL-6 production during inflammatory-induced lung injury has been associated with increased mortality (34). Notably, SOCS3 has been implicated in the regulation of signaling by the IL-6 family of cytokines, through the inhibition of STAT3 activation (35). In the present study, $\mathrm{HG}$ exposure was revealed to potentiate TNF- $\alpha$ and IL-6 levels in A549 cells, whereas treatment with the JAK2/STAT3 inhibitor tyrphostin AG490 attenuated the HG-induced increases in cytokine production. Furthermore, the present findings demonstrated that SOCS3 overexpression similarly prevented the HG-induced upregulation of TNF- $\alpha$ and IL- 6 levels. In addition, the viability of A549 cells was significantly decreased following exposure to $\mathrm{HG}$, indicating the development of $\mathrm{HG}$-induced lung cell injury. These results suggested that SOCS3 may inhibit the HG-induced upregulation of JAK2/STAT3 proteins, adhesion molecules and cytokines in the lungs, thus suggesting a critical role for the SOCS3/JAK2/STAT3 pathway during the inflammatory responses to hyperglycemia.

SOCS proteins are activated by several stimuli and inhibit JAK/STAT signaling in a negative feedback loop involving various mechanisms (36). In agreement with previous data, HG increased the tyrosine phosphorylation of JAK/STAT members in human MCs and HK2 cells (37). HG may induce the transcriptional activation of STAT3. Along with STAT activation, HG transiently induced SOCS expression (21). In the present study, western blot analysis demonstrated that $\mathrm{HG}$ exposure potentiated the expression of SOCS3, JAK2 and STAT3. In addition, HG induced the phosphorylation of JAK2 and STAT3 proteins compared with the control groups, indicating that HG is a potent inducer of both JAK2 and STAT3 tyrosine phosphorylation. The JAK2 specific inhibitor AG490 inhibited the HG-induced p-STAT3 protein expression. The results also suggested that JAK2 serves an important role in HG activation of STAT3. Similarly, SOCS3 overexpression significantly inhibited HG-induced tyrosine phosphorylation JAK2 and STAT3. A549 cells were treated with D-mannitol to confirm that the effects of HG treatment were not a result of hyperosmolarity.

Previous studies have suggested that the inhibition of JAK/STAT signaling through various mechanisms, including JAK2 inhibition, STAT3 knockdown and pharmacological intervention, may counteract $\mathrm{HG}$-induced JAK/STAT activation and prevent the development of $\mathrm{HG}$-associated injury $(25,38)$. In the present study, SOCS3 overexpression was revealed to prevent tyrosine phosphorylation of JAK2 and STAT3 induced by HG in A549 cells, thus suggesting that SOCS3 may protect against HG-induced lung injury through the inhibition of the JAK2/STAT3 pathway to the progression of chronic inflammatory diseases, as previous studies have demonstrated (39-42). Therefore, it may be hypothesized that strategies aiming to upregulate the expression of SOCS proteins in the lungs have potential for the treatment of patients with diabetic lung injury.

In conclusion, the present study demonstrated that $\mathrm{HG}$ exposure increased SOCS3 expression, induced the activation of the JAK2/STAT3 pathway and potentiated the production of proinflammatory cytokines in A549 cells. Furthermore, SOCS3 overexpression and JAK2/STAT3 inhibition attenuated the $\mathrm{HG}$-induced morphological alterations in lung cells, enhanced their viability and suppressed cytokine production. These findings suggested the involvement of the JAK/STAT/SOCS pathway in hyperglycemia-induced cell responses during the development of diabetic lung injury. Further studies are required to elucidate the molecular mechanisms underlying the roles of SOCS3 in the regulation of JAK2/STAT3 signaling and the modulation of inflammatory responses in the lungs.

\section{Acknowledgements}

The present study was supported by the Renmin Hospital Central Laboratory of Wuhan University (Wuhan, China), 
and the National Natural Science Foundation of China (grant nos. 81471844 and 81501648).

\section{References}

1. Klein OL, Krishnan JA, Glick S and Smith LJ: Systematic review of the association between lung function and type 2 diabetes mellitus. Diabet Med 27: 977-987, 2010

2. Wang HJ, Huang YL, Shih YY, Wu HY, Peng CT and Lo WY: MicroRNA-146a decreases high glucose/thrombin-induced endothelial inflammation by inhibiting NAPDH oxidase 4 expression. Mediators Inflamm 2014: 379537, 2014.

3. Hsia CC and Raskin P: Lung involvement in diabetes: Does it matter? Diabetes Care 31: 828-829, 2008.

4. Orasanu G and Plutzky J: The pathologic continuum of diabetic vascular disease. J Am CollCardiol 53 (5 Suppl): S35-S42, 2009.

5. Pradhan AD, Manson JE, Rifai N, Buring JE and Ridker PM: C-reactive protein, interleukin 6 , and risk of developing type 2 diabetes mellitus. JAMA 286: 327-334, 2001

6. Lee YS and Jun HS: Anti-inflammatory effects of GLP-1-based therapies beyond glucose control. Mediators Inflamm 2016 : 3094642, 2016

7. Wang X, Bao W, Liu J, Ouyang YY, Wang D, Rong S, Xiao X, Shan ZL, Zhang Y, Yao P and Liu LG: Inflammatory markers and risk of type 2 diabetes: A systematic review and meta-analysis. Diabetes Care 36: 166-175, 2013.

8. Williams MD and Nadler JL: Inflammatory mechanisms of diabetic complications. Curr Diab Rep 7: 242-248, 2007.

9. Stuart MJ and Baune BT: Depression and type 2 diabetes: Inflammatory mechanisms of a psychoneuroendocrine co-morbidity. Neurosci Biobehav Rev 36: 658-676, 2012.

10. Liu Q, Xing L, Wang L, Yao F, Liu S, Hao J, Liu W and Duan H: Therapeutic effects of suppressors of cytokine signaling in diabetic nephropathy. J Histochem Cytochem 62: 119-128, 2014.

11. Hu C, Sun L, Xiao L, Han Y, Fu X, Xiong X, Xu X, Liu Y, Yang S, Liu F and Kanwar YS: Insights into the mechanisms involved in the expression and regulation of extracellular matrix proteins in diabetic nephropathy. Curr Med Chem 22: 2858-2870, 2015.

12. Marrero MB, Banes-Berceli AK, Stern DM and Eaton DC: Role of the JAK/STAT signaling pathway in diabetic nephropathy. Am J Physiol Renal Physiol 290: F762-F768, 2006.

13. Dalpke A, Heeg K, Bartz H and Baetz A: Regulation of innate immunity by suppressor of cytokine signaling (SOCS) proteins. Immunobiology 213: 225-235, 2008.

14. Inagaki-Ohara $\mathrm{K}$, Kondo $\mathrm{T}$, Ito $\mathrm{M}$ and Yoshimura A: SOCS, inflammation, and cancer. JAKSTAT 2: e24053, 2013.

15. Krebs DL and Hilton DJ: SOCS proteins: Negative regulators of cytokine signaling. Stem Cells 19: 378-387, 2001.

16. Yasukawa H, Sasaki A and Yoshimura A: Negative regulation of cytokine signaling pathways. Annu Rev Immunol 18: 143-164, 2000.

17. Suchy D, Łabuzek K, Machnik G, Kozłowski M and Okopień B: SOCS and diabetes-ups and downs of a turbulent relationship. Cell Biochem Funct 31: 181-195, 2013.

18. Piessevaux J, Lavens D, Peelman F and Tavernier J: The many faces of the SOCS box. Cytokine Growth Factor Rev 19: 371-381, 2008.

19. Yoshimura A, Suzuki M, Sakaguchi R, Hanada T and Yasukawa H: SOCS, inflammation, and autoimmunity. Front Immunol 3: 20, 2012.

20. Sasaki A, Yasukawa H, Suzuki A, Kamizono S, Syoda T, Kinjyo I, Sasaki M, Johnston JA and Yoshimura A: Cytokine-inducible SH2 protein-3 (CIS3/SOCS3) inhibits Janus tyrosine kinase by binding through the $\mathrm{N}$-terminal kinase inhibitory region as well as SH2 domain. Genes Cells 4: 339-351, 1999.

21. Carow B and Rottenberg ME: SOCS3, a major regulator of infection and inflammation. Front Immunol 5: 58, 2014

22. Feng X, Tang H, Leng J and Jiang Q: Suppressors of cytokine signaling (SOCS) and type 2 diabetes. Mol Biol Rep 41: 2265-2274, 2014

23. Alisson-Silva F, Freire-de-Lima L, Donadio JL, Lucena MC, Penha L, Sá-Diniz JN, Dias WB and Todeschini AR: Increase of O-glycosylated oncofetal fibronectin in high glucose-induced epithelial-mesenchymal transition of cultured human epithelial cells. PLoS One 8: e60471, 2013.
24. Huang JS, Chuang LY, Guh JY, Huang YJ and Hsu MS Antioxidants attenuate high glucose-induced hypertrophic growth in renal tubular epithelial cells. Am J Physiol Renal Physiol 293: F1072-F1082, 2007.

25. Wang $X$, Shaw S, Amiri F, Eaton DC and Marrero MB: Inhibition of the Jak/STAT signaling pathway prevents the high glucose-induced increase in tgf-beta and fibronectin synthesis in mesangial cells. Diabetes 51: 3505-3509, 2002

26. Shi Y, Zhang Y, Wang C, Du C, Zhao S, Qi Z, Zhang Q and Duan H: Suppressor of cytokine signaling-1 reduces high glucose-induced TGF-betal and fibronectin synthesis in human mesangial cells. FEBS Lett 582: 3484-3488, 2008.

27. Nakashima T, Yokoyama A, Onari Y, Shoda H, Haruta Y, Hattori N, Naka T and Kohno N: Suppressor of cytokine signaling 1 inhibits pulmonary inflammation and fibrosis. J Allergy Clin Immunol 121: 1269-1276, 2008.

28. Severgnini M, Takahashi S, Tu P, Perides G, Homer RJ, Jhung JW, Bhavsar D, Cochran BH and Simon AR: Inhibition of the Src and Jak kinases protects against lipopolysaccharide-induced acute lung injury. Am J RespirCrit Care Med 171: 858-867, 2005.

29. Ortiz-Muñoz G, Lopez-Parra V, Lopez-Franco O, Fernandez-Vizarra P, Mallavia B, Flores C, Sanz A, Blanco J, Mezzano S, Ortiz A, et al: Suppressors of cytokine signaling abrogate diabetic nephropathy. J Am Soc Nephrol 21: 763-772, 2010.

30. Gao H, Hoesel LM, Guo RF, Rancilio NJ, Sarma JV and Ward PA Adenoviral-mediated overexpression of SOCS3 enhances IgG immune complex-induced acute lung injury. J Immunol 177 612-620, 2006

31. Yan C, Ward PA, Wang X and Gao H: Myeloid depletion of SOCS3 enhances LPS-induced acute lung injury through CCAAT/enhancer binding protein $\delta$ pathway. FASEB J 27: 2967-2976, 2013

32. Qin H, Roberts KL, Niyongere SA, Cong Y, Elson CO and Benveniste EN: Molecular mechanism of lipopolysaccharide-induced SOCS-3 gene expression in macrophages and microglia. J Immunol 179: 5966-5976, 2007.

33. Venieratos PD, Drossopoulou GI, Kapodistria KD, Tsilibary EC and Kitsiou PV: High glucose induces suppression of insulin signalling and apoptosis via upregulation of endogenous IL-1beta and suppressor of cytokine signalling-1 in mouse pancreatic beta cells. Cell Signal 22: 791-800, 2010

34. Goodman RB, Pugin J, Lee JS and Matthay MA: Cytokine-mediated inflammation in acute lung injury. Cytokine Growth Factor Rev 14: 523-535, 2003.

35. Croker BA, Krebs DL, Zhang JG, Wormald S, Willson TA, Stanley EG, Robb L, Greenhalgh CJ, Förster I, Clausen BE, et al: SOCS3 negatively regulates IL-6 signaling in vivo. Nat Immunol 4: 540-545, 2003

36. Alexander WS and Hilton DJ: The role of suppressors of cytokine signaling (SOCS) proteins in regulation of the immune response. Annu Rev Immunol 22: 503-529, 2004.

37. Shi YH, Zhao S, Wang C, Li Y and Duan HJ: Fluvastatin inhibits activation of JAK and STAT proteins in diabetic rat glomeruli and mesangial cells under high glucose conditions. Acta Pharmacol Sin 28: 1938-1946, 2007.

38. Manea SA, Manea A and Heltianu C: Inhibition of JAK/STAT signaling pathway prevents high-glucose-induced increase in endothelin-1 synthesis in human endothelial cells. Cell Tissue Res 340: 71-79, 2010.

39. Hernández-Vargas P, López-Franco O, Sanjuán G, Rupérez M, Ortiz-Muñoz G, Suzuki Y, Aguado-Roncero P, Pérez-Tejerizo G, Blanco J, Egido J, et al: Suppressors of cytokine signaling regulate angiotensin II-activated Janus kinase-signal transducers and activators of transcription pathway in renal cells. J Am SocNephrol 16: 1673-1683,2005.

40. Gómez-Guerrero C, López-Franco O, Sanjuán G, Hernández-Vargas P, Suzuki Y, Ortiz-Muñoz G, Blanco J and Egido J: Suppressors of cytokine signaling regulate Fc receptor signaling and cell activation during immune renal injury. J Immunol 172: 6969-6977, 2004.

41. Suzuki A, Hanada T, Mitsuyama K, Yoshida T, Kamizono S, Hoshino T, Kubo M, Yamashita A, Okabe M, Takeda K, et al: CIS3/SOCS3/SSI3 plays a negative regulatory role in STAT3 activation and intestinal inflammation. J Exp Med 193: 471-481, 2001.

42. Wong PK, Egan PJ, Croker BA, O'Donnell K, Sims NA, Drake S, Kiu H, McManus EJ, Alexander WS, Roberts AW and Wicks IP: SOCS-3 negatively regulates innate and adaptive immune mechanisms in acute IL-1-dependent inflammatory arthritis. J Clin Invest 116: 1571-1581, 2006 